\title{
First-principles calculations of the vacancy formation energy in transition and noble metals
}

Korzhavyi, P.A.; Abrikosov, Igor A.; Johansson, Börje; Ruban, Andrei; Skriver, Hans Lomholt

Published in:

Physical Review B

Link to article, DOI:

10.1103/PhysRevB.59.11693

Publication date:

1999

Document Version

Publisher's PDF, also known as Version of record

Link back to DTU Orbit

Citation (APA):

Korzhavyi, P. A., Abrikosov, I. A., Johansson, B., Ruban, A., \& Skriver, H. L. (1999). First-principles calculations of the vacancy formation energy in transition and noble metals. Physical Review B, 59(18), 11693-11703. https://doi.org/10.1103/PhysRevB.59.11693

\section{General rights}

Copyright and moral rights for the publications made accessible in the public portal are retained by the authors and/or other copyright owners and it is a condition of accessing publications that users recognise and abide by the legal requirements associated with these rights.

- Users may download and print one copy of any publication from the public portal for the purpose of private study or research.

- You may not further distribute the material or use it for any profit-making activity or commercial gain

- You may freely distribute the URL identifying the publication in the public portal 


\title{
First-principles calculations of the vacancy formation energy in transition and noble metals
}

\author{
P. A. Korzhavyi, * I. A. Abrikosov, and B. Johansson \\ Condensed Matter Theory Group, Department of Physics, Uppsala University, S-75121 Uppsala, Sweden \\ A. V. Ruban and H. L. Skriver \\ Center for Atomic-Scale Materials Physics and Department of Physics, Technical University of Denmark, DK-2800 Lyngby, Denmark
}

(Received 23 April 1998; revised manuscript received 22 December 1998)

\begin{abstract}
The vacancy formation energy and the vacancy formation volume of the $3 d, 4 d$, and $5 d$ transition and noble metals have been calculated within the local-density approximation. The calculations employ the order- $N$ locally self-consistent Green's-function method in conjunction with a supercell approach and include electrostatic multipole corrections to the atomic sphere approximation. The results are in excellent agreement with available full-potential calculations and with the vacancy formation energies obtained in positron annihilation measurements. The variation of the vacancy formation energy through a transition-metal series and the effects of crystal and magnetic structure are investigated and discussed. [S0163-1829(99)07717-6]
\end{abstract}

\section{INTRODUCTION}

The existence of vacancies in metals and intermetallic compounds plays an important role for the kinetic and thermodynamic properties of materials. In this connection the energy of formation of a monovacancy is a key concept in the understanding of the processes that occur in alloys during mechanical deformation or heat treatment. Over the last decade studies have succeeded in determining energies of monovacancy formation from positron annihilation experiments $^{1-3}$ as well as from $a b$ initio full-potential (FP) calculations for simple metals ${ }^{4-6}$ and for some transition and noble metals. ${ }^{7-10}$ The most recent work even includes studies of vacancy-vacancy and vacancy-solute interactions in $\mathrm{Cu}$, $\mathrm{Ni}, \mathrm{Ag}$, and Pd (Refs. 11-14) and in Al (Ref. 15) by means of the full-potential Korringa-Kohn-Rostoker (KKR) Green's-function method.

In spite of the importance, to our knowledge, no $a b$ initio study of the behavior of the vacancy formation energy along a transition-metal series has been performed so far, although a number of theoretical predictions based on simplified models of bonding in transition metals may be found in the literature. ${ }^{16-18}$ On the other hand, there are many experimentally observed and theoretically expected correlations connecting the vacancy formation energy to other physical properties such as cohesive and surface energies, ${ }^{9,10,19-21}$ melting and Debye temperatures, ${ }^{3,22,23}$ and elastic constants. ${ }^{24}$ Therefore, the goals of the present work are to perform a systematic $a b$ initio study of vacancy formation energies in all the $3 d, 4 d$, and $5 d$ transition metals, to analyze the observed trends along the rows of the Periodic Table, and to investigate the effects of crystal and magnetic structure on the vacancy formation energies.

The number of vacancies in an elemental metal at equilibrium conditions is usually small. Even close to the melting point it is much less than 1 at. \%. Thus, in order to study a realistic metal-vacancy system, it is necessary to consider either a single vacancy in the bulk using the Green'sfunction method, ${ }^{7,8}$ or a large supercell in which the vacancies are well separated. To calculate vacancy-vacancy or vacancy-solute interaction energies in alloys or to study complex thermal defects in some intermetallics, very large supercells are necessary. Since the computational effort in conventional band-structure methods grows as $N^{3}$, where $N$ is the number of atoms in the unit cell, such methods become extremely inefficient for large supercells. However, recently a new generation of electronic structure methods in which the computer time scales linearly with $N$ has emerged. ${ }^{25-28}$ One most efficient so-called order- $N$ method is the locally self-consistent Green's-function (LSGF) method by Abrikosov et al. ${ }^{27,28}$ that may be used to treat ordered, disordered, and partially ordered systems on the same footing and with an accuracy comparable to that of conventional bandstructure methods. The high efficiency of this method is achieved by the choice of a self-consistent effective medium that allows for a particularly small local interaction zone (LIZ).

The original implementation of the LSGF method ${ }^{27}$ is based on the atomic-sphere approximation (ASA) that may be expected to overestimate the vacancy formation energy by as much as a factor of 2 similar to all the previous vacancy calculations performed within the ASA. ${ }^{29-31}$ This large error arises as a result of an inadequate treatment of the electron charge depletion around the vacancy caused by the spherical averaging of the electron density over each atomic sphere and would clearly invalidate the calculations. However, the vacancy formation energy is a physical quantity of the same kind (and order of magnitude) as the surface energy and one would expect to be able to determine it by means of the LSGF method provided the spherical approximation to the electron charge density is lifted ${ }^{7,8}$ similar to the surface energy calculations for simple and transition metals ${ }^{32}$ and to the surface segregation calculations in metallic alloys. ${ }^{33,34}$ In the present work we therefore keep the spherically symmetric potential but go beyond the ASA for the electron density by an electrostatic multipole expansion and we show that in this formulation the LSGF method has the accuracy necessary to calculate the vacancy formation energy in transition metals. 


\section{METHOD OF CALCULATIONS}

\section{A. Thermodynamic definitions}

Let us consider a bulk crystal of an elemental metal containing $N_{a}$ atoms and $N_{v}$ vacancies at zero temperature. The number of vacancies in the system is allowed to change while the number of atoms is kept constant. We then define the vacancy concentration as $c_{v}=N_{v} / N$, where $N=N_{a}$ $+N_{v}$ is the total number of lattice sites and evaluate the vacancy formation quantities in the dilute limit $c_{v} \rightarrow 0$.

At constant pressure $p$, corresponding to the experimental conditions, the vacancy formation enthalpy may be defined as

$$
H_{1 V}^{F}=\left(\left.\frac{\partial E\left(N_{v}\right)}{\partial N_{v}}\right|_{N_{v}=0}\right)_{p, N_{a}}+p\left(\left.\frac{\partial V\left(N_{v}\right)}{\partial N_{v}}\right|_{N_{v}=0}\right)_{p, N_{a}},
$$

where $E\left(N_{v}\right)$ is the total energy and $V\left(N_{v}\right)$ the volume of the system. The pressure dependence of the vacancy formation enthalpy is commonly characterized by the first derivative at zero pressure, the so-called vacancy formation volume,

$$
\Omega_{1 V}^{F}=\left.\frac{\partial H_{1 V}^{F}}{\partial p}\right|_{p=0}=\left.\left(\Omega_{0}+\frac{\partial \Omega_{0}}{\partial c_{v}}\right)\right|_{c_{v}=0},
$$

where $\Omega_{0}=V_{0}\left(c_{v}\right) / N$ is the equilibrium volume per site. At constant volume $V$ the vacancy formation energy may be defined as

$$
E_{1 V}^{F}=\left(\left.\frac{\partial E\left(N_{v}\right)}{\partial N_{v}}\right|_{N_{v}=0}\right)_{V, N_{a}} .
$$

The vacancy formation energy, Eq. (3), evaluated at the calculated equilibrium volume $V_{0}$ and the vacancy formation enthalpy, Eq. (1), determined at zero external pressure coincide.

The volume dependence of the vacancy formation energy may be described by a partial contribution to the crystal pressure, ${ }^{35}$

$$
p_{1 V}=\left(\left.\frac{\partial p}{\partial c_{v}}\right|_{c_{v}=0}\right)_{\Omega_{0}}=-\left.\frac{\partial E_{1 V}^{F}(\Omega)}{\partial \Omega}\right|_{\Omega=\Omega_{0}},
$$

connected to the vacancy formation volume (2) through the thermodynamic relationship

$$
p_{1 V}=B\left(\frac{\Omega_{1 V}^{F}}{\Omega_{0}}-1\right)=B \delta
$$

where $B$ is the bulk modulus of the host and $\delta$ is the volume misfit

$$
\delta=\left.\frac{1}{\Omega_{0}} \frac{\partial \Omega_{0}}{\partial c_{v}}\right|_{c_{v}=0}
$$

\section{B. Supercell calculations for vacancies}

To calculate the physical quantities relevant for vacancy formation we use a supercell approach in which the metalvacancy system is modeled by a bulk crystal in which the number of lattice sites $N=N_{a}+N_{v}$ is fixed by construction. Following the definitions (1) and (2) the vacancy formation enthalpy and the vacancy formation volume may be calculated from the expressions

$$
H_{1 V}^{F}=E(1, \widetilde{\Omega})-\frac{N-1}{N} E(0, \Omega)+p[N \widetilde{\Omega}-(N-1) \Omega]
$$

and

$$
\Omega_{1 V}^{F}=N \widetilde{\Omega}_{0}-(N-1) \Omega_{0},
$$

where $E\left(N_{v}, \widetilde{\Omega}\right)$ is the total energy of a supercell containing $N_{v}$ vacancies at a volume $\widetilde{\Omega}$ corresponding to an external pressure $p$. For the perfect crystal with no vacancies present the volume corresponding to the common external pressure is $\Omega$.

If the size of the supercell is large, the effect of volume relaxation on the energy is negligible since the total energy of the supercell has a minimum at the appropriate equilibrium volumes. In that case the vacancy formation energy may be calculated at a fixed volume close to the equilibrium volume $\Omega_{0}$ as

$$
E_{1 V}^{F}(\Omega)=E(1, \Omega)-\frac{N-1}{N} E(0, \Omega),
$$

while the partial vacancy pressure may be estimated from its first derivative using Eq. (4). Due to the superior convergence of Eq. (7) as $N \rightarrow \infty$, we have used this expression at zero external pressure instead of Eq. (9) to calculate the vacancy formation energy as is common practice in supercell calculations for vacancies. $5,10,30,36$

\section{Locally self-consistent Green's-function method}

To eliminate vacancy-vacancy interactions one needs supercells with about 30 to 50 lattice sites and since we want to perform total-energy calculations for close to 30 elemental metals in two crystal structures, bcc and fcc, we will here take advantage of the LSGF method, ${ }^{27,28}$ the computational efforts of which scale linearly with the number of lattice sites. This favorable order- $N$ scaling is achieved by solving the multiple-scattering problem exactly inside the so-called local interaction zone that surrounds each atom in the supercell whereby one may obtain locally self-consistent values of the Green's function for the central site of each LIZ. The LSGF method employs a judiciously chosen effective medium to describe the crystal beyond the LIZ and therefore one may obtain highly accurate total energies for a minimal LIZ size. In fact, the method typically becomes more efficient than conventional order- $N^{3}$ methods for supercells with more than 20 atoms and is ideally suited for the present purpose. A detailed description of the method and some applications may be found in Ref. 28 .

The LSGF method is based on the linear muffin-tin orbitals (LMTO) theory of Andersen ${ }^{37-42}$ and employs the ASA in which one includes only the electrostatic monopole con- 
tribution to the atomic-centered spherically symmetric potentials. This approximation has proved to be accurate for highly symmetric and close-packed systems, such as ordinary metallic crystals, but the neglect of higher multipoles makes the ASA fail, for instance, in surface calculations. For the same reason Braun et al. ${ }^{30}$ find vacancy formation energies for $\mathrm{Fe}$ that in the ASA are 2.5 times larger than the experimental value. Clearly, to obtain reliable vacancy formation energies one needs to go beyond the ASA.

\section{Corrections to the ASA}

Similar to the LMTO Green's-function method for surface calculations ${ }^{32}$ we include in the present work the monopole contribution to the ASA potential from the higher multipoles of the charge density, i.e., go beyond the ASA for the charge density but keep the ASA for the potential. For want of a better name we call this approximation, which is the first step towards the full charge-density technique, ${ }^{43}$ ASA $+\mathrm{M}$.

The monopole $(L=s)$ contribution to the effective oneelectron potential is evaluated from the monopole and multipole moments, $Q_{\mathbf{R}}^{L}$, of the valence electron charge by the multipole expansion

$$
V_{\mathbf{R}}^{s}=\frac{1}{S} \sum_{\mathbf{R}^{\prime}, L^{\prime}} M_{\mathbf{R}, \mathbf{R}^{\prime}}^{s, L^{\prime}} \quad Q_{\mathbf{R}^{\prime}}^{L^{\prime}},
$$

where $L$ is shorthand for the $(l, m)$ quantum numbers and $M_{\mathbf{R}, \mathbf{R}^{\prime}}^{L, L^{\prime}}$ is the multipole Madelung matrix that is equivalent to the conventional (unscreened, $\alpha=0$ ) LMTO structure constants for the entire supercell. A corresponding Madelung contribution given by

$$
E_{M}=\frac{1}{2 S} \sum_{\mathbf{R}, L} Q_{\mathbf{R}}^{L} \sum_{\mathbf{R}^{\prime}, L^{\prime}} M_{\mathbf{R}, \mathbf{R}^{\prime}}^{L, L^{\prime}} Q_{\mathbf{R}^{\prime}}^{L^{\prime}}
$$

is added to the total energy. A description of the procedure including expressions for the Madelung matrices and the multipole moments may be found in Ref. 32 where it is also shown that only when the multipole contributions to the ASA potential are included does one obtain accurate surface energies.

The number of multipoles included in the $L$ summations in Eqs. (10) and (11) is determined by the angular momentum cutoff $l_{\max }$ used in the Green's-functions calculations. In the present calculations $l_{\text {max }}=3$ and due to the properties of the Gaunt coefficients the multipole moments of the charge density have nonzero components up to $l=2 l_{\text {max }}$. As a result the Madelung contributions to the potential and total energy include angular momenta up to $l=6$.

In Table I we show the monopole Madelung potential for the atomic spheres at and around a vacancy in $\mathrm{Cu}$ as a function of $l_{\text {max }}$ together with the corresponding self-consistent net charge and contribution to the total energy. It is seen that the multipole terms lower the potential by $30 \%$ and lead to a substantial reduction of the Madelung energy. In fact, this reduction brings the calculated vacancy formation energy for $\mathrm{Cu}$ in complete agreement with the results of full-potential calculations. $^{13}$

As a minor correction we also include the muffin-tin ${ }^{44}$ or Ewald $^{45}$ correction to the ASA Madelung energy
TABLE I. Madelung potential, net charge, and partial Madelung energy of the atomic spheres belonging to the $i$ th coordination shell around the vacancy site in fcc $\mathrm{Cu}$ for $R_{W S}=2.58$ bohrs calculated using a 32-site supercell without $\left(l_{\max }=0\right)$ and with $\left(l_{\max }=6\right)$ multipole Madelung terms. The vacancy site is at $i=0$ and the LIZ size is two coordination shells.

\begin{tabular}{lccrr}
\hline \hline $\begin{array}{l}\text { Coordination } \\
\text { shell number }\end{array}$ & $\begin{array}{c}\text { Multipole } \\
l_{\max }\end{array}$ & $\begin{array}{c}V_{R}^{s}(\mathbf{R}) \\
(\mathrm{Ry})\end{array}$ & $\begin{array}{c}Q_{R}^{s}(\mathbf{R}) \\
(|e|)\end{array}$ & \multicolumn{1}{c}{$\begin{array}{c}E_{M}(\mathbf{R}) \\
(\mathrm{Ry} / \mathrm{site})\end{array}$} \\
\hline 0 & 0 & -0.4387 & 0.9022 & -0.1979 \\
& 6 & -0.6495 & 1.0299 & -0.3346 \\
1 & 0 & 0.0897 & -0.0873 & -0.0039 \\
& 6 & 0.0797 & -0.0894 & -0.0078 \\
2 & 0 & -0.0120 & -0.0083 & 0.0000 \\
& 6 & -0.0097 & 0.0003 & 0.0000 \\
\hline \hline
\end{tabular}

$$
E_{M}^{c o r r}=\frac{1}{2} e^{2}\left(1.8-\alpha_{M}\right) \frac{\langle q(S)\rangle^{2}}{S} .
$$

Here, $e$ is the electron charge ( $e^{2}=2$ in Rydberg units), $S$ the radius of the atomic sphere (it is assumed that all the atomic spheres have equal radii), and $\alpha_{M}$ the Madelung constant for the underlying lattice. The effective charge $\langle q(S)\rangle$ is the average interstitial electron density in the crystal multiplied by the atomic sphere volume. In the present case it becomes

$$
\langle q(S)\rangle=\frac{4}{3} \pi S^{3} \frac{1}{N} \sum_{\mathbf{R}} n_{\mathbf{R}}(S),
$$

where the summation runs over all of the $N$ atomic positions $\mathbf{R}$ of the supercell.

For closed-packed underlying lattices $\alpha_{M}$ is close to 1.8, which is the value of $\alpha_{M}$ in the ASA, and for most metals at their equilibrium volume, the Ewald correction is typically in the range from 10 to $40 \mathrm{mRy} / \mathrm{atom}$. The correction has been included in a number of calculations, ${ }^{46-48}$ where it tends to increase the calculated equilibrium lattice parameter that is usually underestimated within the ASA. The importance of this term in the vacancy problem comes from two facts. First, the vacancy formation energy is very sensitive to the atomic volume and, second, a vacancy lowers the interstitial electron density that enters Eq. (12). The latter effect is much stronger than in normal alloys, say, between two transition metals, which have $n_{\mathbf{R}}(S)$ of the same order for all the alloy components.

\section{E. Convergence with respect to LIZ and supercell size}

To test the convergence of the LSGF in the ASA $+\mathrm{M}$ we have calculated the vacancy formation energy in $\mathrm{Cu}$ varying the LIZ and supercell sizes. The result of this convergence test is summarized in Table II. In general we find that the net charge of the vacancy, i.e., the empty sphere, shows an attenuated oscillatory behavior with increasing LIZ size, that the Madelung shift on the vacant site converges monotonously, and that the resulting value of the vacancy formation energy is insensitive to an increase of the LIZ size beyond the first coordination shell (1 c.s.). The difference between the vacancy formation energies calculated using a 32-site or a 108 -site $(3 \times 3 \times 3)$ fcc supercell is found to be $0.01 \mathrm{eV}$. 
TABLE II. Net charge inside the empty sphere, Madelung potential and energy of the vacancy site, and the vacancy formation energy in $\mathrm{Cu}$ calculated using different supercell and LIZ sizes.

\begin{tabular}{lcccccc}
\hline \hline $\begin{array}{l}\text { Supercell } \\
\text { size }\end{array}$ & $\begin{array}{c}\text { LIZ } \\
\text { size }\end{array}$ & $\begin{array}{c}\text { Multipole } \\
l_{\max }\end{array}$ & $\begin{array}{c}Q_{v a c}^{s} \\
(|e|)\end{array}$ & $\begin{array}{c}V_{v a c}^{s} \\
(\mathrm{Ry})\end{array}$ & $\begin{array}{c}E_{M}^{v a c} \\
(\mathrm{Ry} / \mathrm{site})\end{array}$ & $\begin{array}{c}H_{1 V}^{F} \\
(\mathrm{eV})\end{array}$ \\
\hline 32 & 2 c.s. & 0 & 0.9022 & -0.4387 & -0.1979 & 2.90 \\
32 & 1 c.s. & 6 & 1.0312 & -0.6504 & -0.3354 & 1.33 \\
32 & 2 c.s. & 6 & 1.0299 & -0.6495 & -0.3346 & 1.33 \\
32 & 3 c.s. & 6 & 1.0306 & -0.6491 & -0.3346 & 1.33 \\
108 & 2 c.s. & 6 & 1.0312 & -0.6403 & -0.3302 & 1.32 \\
\hline \hline
\end{tabular}

This indicates that the interaction of vacancies at long distances is repulsive and rather small.

As a further indication of the accuracy of the method we have calculated the formation energy of divacancies in $\mathrm{Cu}$ using 64-site $(2 \times 2 \times 4)$ supercells in which the two vacancies are first and second neighbors, respectively. The maximum separation between the vacancies in a 64-site supercell corresponds exactly to the separation of the vacancies in a 32-site supercell with a single vacancy, and this allows us to extract the vacancy-vacancy interactions in $\mathrm{Cu}$. Our calculated divacancy binding energy is shown in Table III and it compares well the results of the full-potential studies. ${ }^{13}$

It is seen that the interaction between two vacancies is small and attractive when they are first neighbors but repulsive when they are second neighbors. Despite the fact that the divacancy binding energy is of the same order of magnitude as the energy of the local lattice relaxations around a single vacancy, the relaxation effects cancel to a large degree in the final expression for the binding energy of a divacancy, and the residual contribution to the divacancy binding energy due to local relaxation effects should be one order of magnitude smaller.

\section{F. Details of calculations}

Based on the convergence tests we have used the LSGF method $^{27}$ in conjunction with the ASA $+\mathrm{M}$ to performed scalar-relativistic total-energy calculations of monovacancies in the $3 d, 4 d$, and $5 d$ transition metals. For the magnetic $3 d$ metals we included the effect of spin polarization. To analyze the variation of the vacancy formation energy along the rows of the Periodic Table all the metals were calculated in the fcc as well as bcc crystal structures. We assumed equal atomic radii for metal atoms and vacancies and included an-

TABLE III. The monovacancy formation energy, $H_{1 V}^{F}$, and the binding energies of two vacancies which are first, $E_{2 V}^{B}(1)$, and second, $E_{2 V}^{B}(2)$, neighbors in fcc $\mathrm{Cu}$. All energies are given in $\mathrm{eV}$. Negative sign of the binding energy means attraction. LSGF $\mathrm{ASA}+\mathrm{M}$ refers to the present calculations for a 32-site supercell with one vacancy and a 64-site supercell with two vacancies. The results of the full-potential calculations by Klemradt et al. (Ref. 13) are labeled FP-KKR-GF.

\begin{tabular}{lccc}
\hline \hline & $H_{1 V}^{F}$ & $E_{2 V}^{B}(1)$ & $E_{2 V}^{B}(2)$ \\
\hline LSGF ASA+M & 1.33 & -0.096 & 0.080 \\
FP-KKR-GF & 1.41 & -0.076 & \\
\hline \hline
\end{tabular}
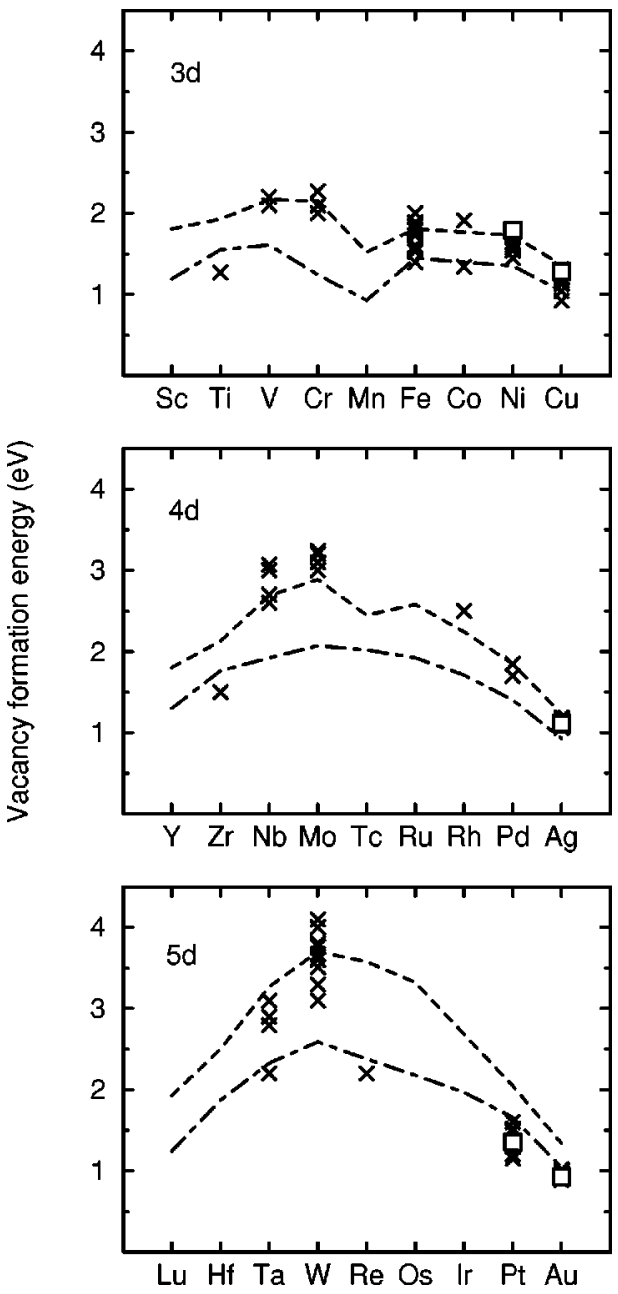

FIG. 1. Experimental data for the monovacancy formation energies in the $3 d, 4 d$, and $5 d$ transition and noble metals (crosses). Recommended medium-temperature values are shown as open squares. The broken line represents the vacancy formation energies given by the $T_{M} / 1000$ rule, while the dot-dashed line shows the vacancy formation energies predicted by Miedema's theory.

gular momenta up to $l_{\text {max }}=3$. Thus, we treated valence $s, p$, $d$, and $f$ electrons self-consistently within the local-density approximation for which we used the Perdew, Burke, and Ernzerhof $^{49}$ parametrization of the many-body results by Ceperley and Alder ${ }^{50}$ for the nonmagnetic metals and for ferromagnetic bcc Fe, fcc $\mathrm{Co}$, and $\mathrm{Ni}$ and antiferromagnetic fcc $\mathrm{Mn}$ and bcc $\mathrm{Cr}$.

The core states were recalculated at each self-consistency loop using the soft-core approximation. Semicore states of $\mathrm{Sc}, \mathrm{Ti}, \mathrm{Y}, \mathrm{Zr}$, and Hf were treated using two energy panels, while $\mathrm{Lu}$ was calculated in the frozen core approximation to avoid problems with its $4 f$ states.

The Brillouin zone integration was performed by means of the special point technique, including $240 \mathbf{k}$ points in the $1 / 48$ irreducible wedge of the Brillouin zone for the fcc lattice and 285 points for the bcc lattice. The moments of the state density were evaluated by a 16-point Gaussian integration on a complex energy contour enclosing the occupied states.

Test calculations for 32- and 108-site fcc supercells show that a 32-site supercell is sufficient to calculate vacancy formation energy in the fcc structure. This follows from the fact 
TABLE IV. Vacancy formation energy in $\mathrm{eV}$ for the transition metals in the fcc and bcc crystal structures. LSGF ASA $+M$ refers to the present calculations and FP to previous full-potential calculations.

\begin{tabular}{|c|c|c|c|c|}
\hline \multirow[t]{2}{*}{ Metal } & \multicolumn{2}{|c|}{ fcc } & \multicolumn{2}{|c|}{ bcc } \\
\hline & $\mathrm{LSGF} \mathrm{ASA}+\mathrm{M}$ & FP & LSGF ASA $+\mathrm{M}$ & FP \\
\hline Sc & 1.52 & $1.85^{\mathrm{a}}$ & 1.21 & \\
\hline $\mathrm{Ti}$ & 1.67 & $2.13^{\mathrm{a}}$ & 1.63 & \\
\hline $\mathrm{V}$ & 2.03 & & 2.20 & $3.06^{\mathrm{b}}$ \\
\hline $\operatorname{Cr}(p)$ & 2.12 & & 2.70 & $2.86^{\mathrm{b}}$ \\
\hline $\operatorname{Cr}(a)$ & & & 2.80 & \\
\hline $\operatorname{Mn}(p)$ & 2.51 & & 1.51 & \\
\hline $\operatorname{Mn}(f)$ & 2.51 & & & \\
\hline $\mathrm{Fe}(p)$ & 2.65 & & 1.30 & \\
\hline $\operatorname{Fe}(f)$ & & & 2.25 & \\
\hline $\operatorname{Co}(p)$ & 2.18 & $2.18^{\mathrm{a}}$ & 1.52 & \\
\hline $\operatorname{Co}(f)$ & 2.15 & & & \\
\hline $\mathrm{Ni}(p)$ & 1.67 & $1.76,^{\mathrm{a}} 1.77^{\mathrm{b}}$ & 1.65 & \\
\hline $\mathrm{Ni}(f)$ & 1.78 & & & \\
\hline $\mathrm{Cu}$ & 1.33 & $\begin{array}{c}1.41,{ }^{\mathrm{a}} 1.33^{\mathrm{b}} \\
1.29^{\mathrm{c}}\end{array}$ & 1.23 & \\
\hline $\mathrm{Y}$ & 1.51 & $1.74^{\mathrm{a}}$ & 1.12 & \\
\hline $\mathrm{Zr}$ & 1.70 & $1.77^{\mathrm{a}}$ & 1.68 & \\
\hline $\mathrm{Nb}$ & 2.00 & & 2.32 & $2.92^{\mathrm{b}}$ \\
\hline Mo & 2.17 & & 2.50 & $3.13,^{\mathrm{b}} 2.90^{\mathrm{d}}$ \\
\hline $\mathrm{Tc}$ & 2.46 & $2.56^{\mathrm{a}}$ & 1.45 & \\
\hline $\mathrm{Ru}$ & 2.76 & $2.78^{\mathrm{a}}$ & 1.02 & \\
\hline $\mathrm{Rh}$ & 2.16 & $2.08,{ }^{\mathrm{a}} 2.26^{\mathrm{c}}$ & 1.32 & \\
\hline $\mathrm{Pd}$ & 1.43 & $1.57,{ }^{\mathrm{a}} 1.65^{\mathrm{b}}$ & 1.43 & \\
\hline $\mathrm{Ag}$ & 0.96 & $\begin{array}{c}1.20,^{\mathrm{a}} 1.24^{\mathrm{b}} \\
1.06^{\mathrm{c}}\end{array}$ & 0.87 & \\
\hline $\mathrm{Lu}$ & 1.54 & & 1.08 & \\
\hline $\mathrm{Hf}$ & 1.69 & & 1.56 & \\
\hline $\mathrm{Ta}$ & 2.42 & & 2.41 & $3.49^{\mathrm{b}}$ \\
\hline $\mathrm{W}$ & 2.49 & & 3.04 & $3.27^{\mathrm{b}}$ \\
\hline $\operatorname{Re}$ & 2.89 & & 1.27 & \\
\hline Os & 3.19 & & 0.66 & \\
\hline Ir & 2.27 & & 0.68 & \\
\hline $\mathrm{Pt}$ & 1.21 & $1.45^{\mathrm{b}}$ & 1.42 & \\
\hline $\mathrm{Au}$ & 0.77 & $0.82^{b}$ & 0.81 & \\
\hline
\end{tabular}

${ }^{\mathrm{a}}$ FP-KKR-GF, Refs. 7 and 8.

${ }^{\mathrm{b}}$ FP-LMTO, Ref. 10.

${ }^{\mathrm{c}}$ FP-LMTO, Ref. 9.

${ }^{\mathrm{d}}$ Pseudopotential, Ref. 36.

that the total-energy change caused by vacancy-vacancy interaction, see Table III, is one order of magnitude smaller than the vacancy formation energy even when two vacancies are nearest neighbors, and decreases further with increasing distance. For the bcc structure the difference in vacancy formation energies calculated using a 27 -site $(3 \times 3 \times 3$ rhombohedral) and a 54 -site $(3 \times 3 \times 3$ cubic) supercell was found to be larger, e.g., $0.03 \mathrm{eV}$ for $\mathrm{Cr}, 0.12 \mathrm{eV}$ for $\mathrm{Mo}$, and 0.18 $\mathrm{eV}$ for $\mathrm{W}$, and therefore we used the 54-site supercells in this case.

The local interaction zone was chosen to contain two coordination shells around each atom for the fcc supercells and three coordination shells for the supercells with an underlying bcc lattice. Further increase of the LIZ size did not lead to any significant change in the vacancy formation energy.
Thus, for fcc $\mathrm{Cu}$ the difference was found to be less than $0.01 \mathrm{eV}$ (see Table II). The Madelung potential and energy were calculated by means of Eqs. (10) and (11) with summation up to $l=6$.

For each pure metal and metal-vacancy system we performed three self-consistent calculations at three different lattice parameters close to the equilibrium. The total energies at equilibrium were calculated using a parabolic fit to the results of the self-consistent calculations. Thus, volume relaxation of the crystal lattice was taken into account in the calculations of the vacancy formation energies although, of course, its effect was very small especially in the case of the 54-site supercell. The effect of local lattice relaxations that is known to be relatively small for vacancies in transition metals $^{7,8,51,36}$ was neglected. 

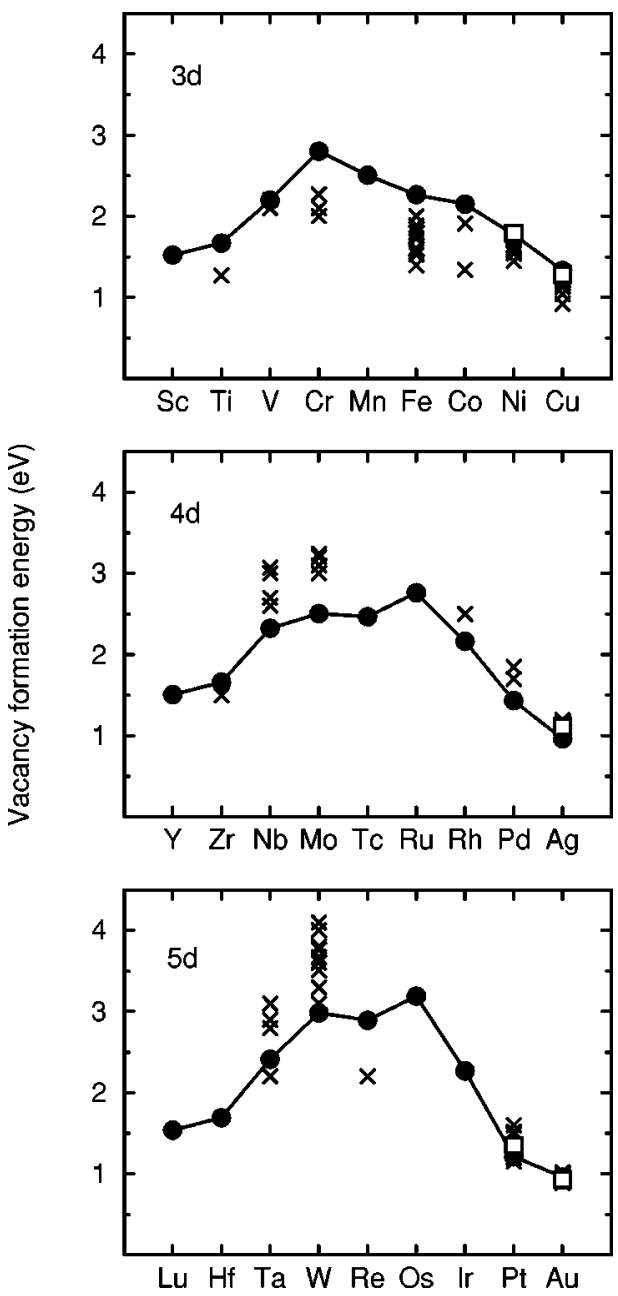

FIG. 2. Calculated monovacancy formation energies for the $3 d$, $4 d$, and $5 d$ transition and noble metals (filled circles connected by a solid line) compared with the experimental results of Fig. 1. The fcc and bcc metals have been treated in their low-temperature crystal structures while Mn and the hcp metals have been treated as fcc. For the magnetic $3 d$ metals the calculations were spin polarized.

\section{MONOVACANCY FORMATION ENERGY}

\section{A. Experimental results}

Before we present the results of our calculations it is useful to analyze the experimental data, and to this end we show in Fig. 1 most of the available experimental vacancy formation energies for the three transition-metal series. In the figure one immediately observes a substantial scatter in the data and it appears that in some important cases, such as Fe, the results obtained using different experimental techniques vary too much to be useful in a quantitative comparison with firstprinciples calculations. For Fe, part of the uncertainty stems from the strong interaction of the vacancies with carbon impurities. ${ }^{52}$ Similarly, problems with sample purity prevents the determination of the vacancy formation energy in Ir. $^{3}$ However, recently the situation has improved and based on positron annihilation experiments a consensus has been reached as to the vacancy formation energies in a number of transition metals, ${ }^{2}$ and these have been tabulated as "recommended values for medium temperatures" with estimated errors of $\pm 0.05 \mathrm{eV}^{3}$

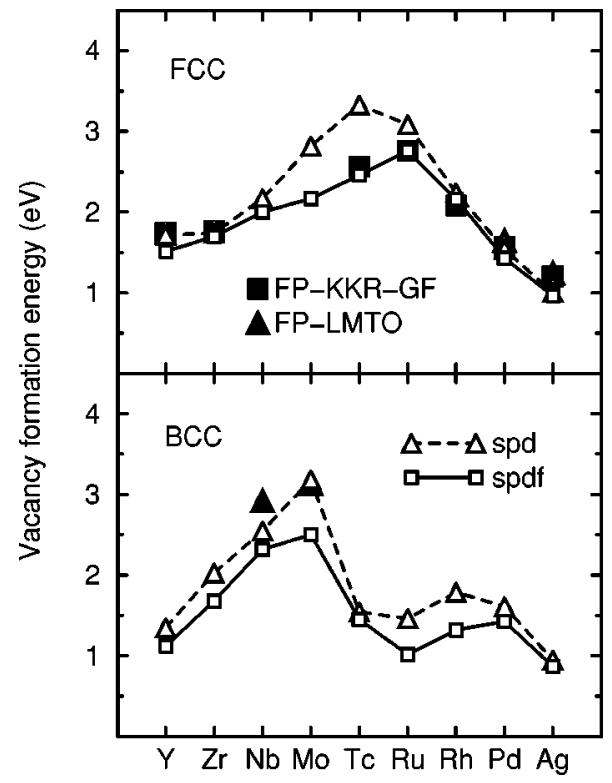

FIG. 3. The monovacancy formation energies for the $4 d$ metals calculated in the two cubic crystal structures and compared with available full-potential calculations. The present calculations were performed with two different sets of basis functions so as to compare with previous full-potential results. The captions in the figure apply to both panels.

In the past Miedema's "macroscopic atom" mode 17 has been fitted to the experimental data and the result of this procedure is shown in Fig. 1 as the dot-dashed line. The values given by the Miedema theory are sometimes even referred to as experimental data, ${ }^{18}$ although they only represent a fit to a very limited number of the old experimental data, as may be seen in the figure. A similar fit based on the empirical correlation with the melting temperature $T_{M}$ in the form $H_{1 V}^{F}=T_{M} / 1000$, where the value of the denominator of $1000 \mathrm{~K} / \mathrm{eV}$ has been determined on the basis of the new positron annihilation data. ${ }^{3}$ The $T_{M} / 1000$ rule has been shown to work well not only for transition metals ${ }^{3}$ and can therefore be used in a few cases in lieu of experimental data.

\section{B. Theoretical results}

The calculated zero-temperature vacancy formation energies [Eq. (7)] for the $3 d, 4 d$, and $5 d$ transition and noble metals are presented in Table IV and Fig. 2 together with experimental data including the recommended values. In the calculations all the metals have been treated in their lowtemperature equilibrium crystal structure except for $\mathrm{Mn}$ and the hcp metals that were treated as fcc. For the magnetic $3 d$ metals we included the effect of spin poplarization. As the figure shows, the trends are well described by the calculations and in those cases where recommended experimental data exist the agreement between theory and experiment is excellent.

As seen in Fig. 2 there exist a number of cases, notably $\mathrm{Ti}, \mathrm{Cr}, \mathrm{Fe}, \mathrm{Nb}, \mathrm{Mo}$, and $\mathrm{Re}$, where the agreement between theory and experiment is rather poor. To determine whether these discrepancies may be a result of the approximations in our approach, we compare in Fig. 3 the present vacancy formation energies with those obtained in previous full- 

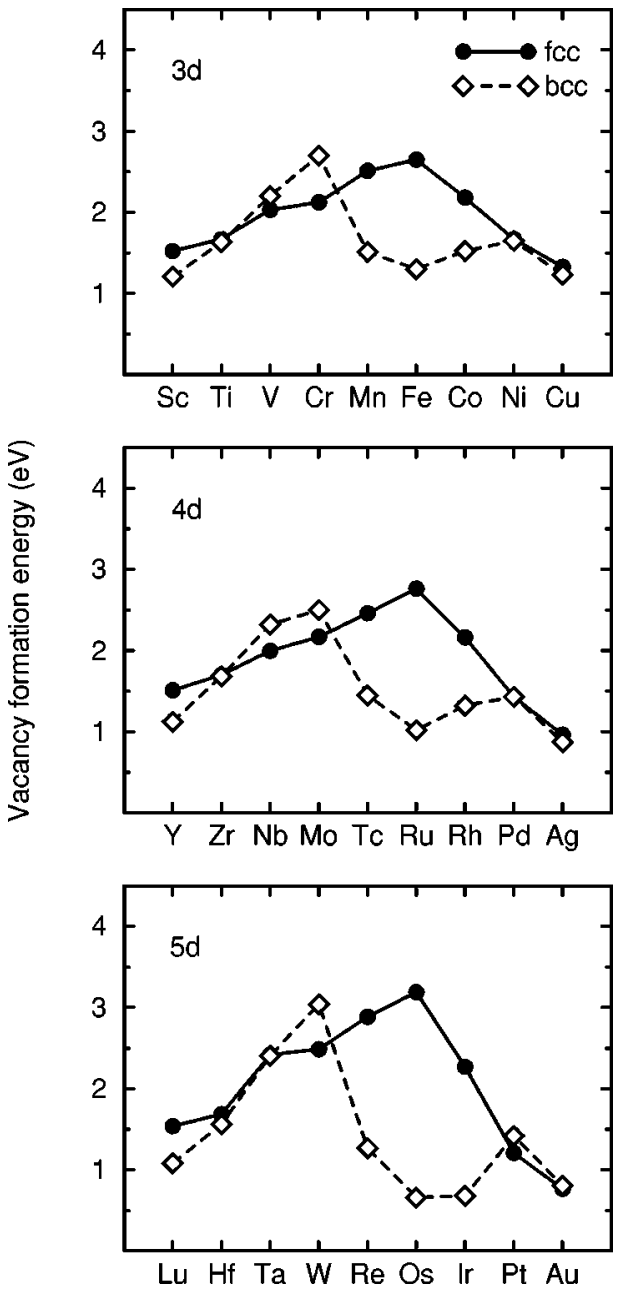

FIG. 4. The monovacancy formation energies for the $3 d, 4 d$, and $5 d$ transition and noble metals calculated in the fcc and bcc structures showing the effect of the crystal structure. All calculations including those for the magnetic $3 d$ metals were non-spinpolarized.

potential calculations. ${ }^{7-10}$ From this comparison we find that provided we include $s, p, d$, and $f$ orbitals in our calculations the agreement with the full-potential KKR Green's-function results ${ }^{7,8}$ is excellent for all the fcc $4 d$ metals. In contrast, the agreement with the FP-LMTO results of Korhonen et al. ${ }^{10}$ for the bcc $4 d$ metals is unsatisfactory except when we use the $s p d$ basis also used in the FP-LMTO calculations. We conclude that the present approach including an spdf basis yields vacancy formation energies with an accuracy similar to that obtained in the most accurate full-potential methods.

\section{General trends}

For a transition-metal series the vacancy formation energy plotted as a function of the $d$ occupation number exhibits roughly a parabolic variation with a maximum close to the middle of the series. This is similar to the more well-known cases of the cohesive and surface energies that are usually explained using Friedel's rectangular state-density model in the tight-binding approximation. ${ }^{53}$ When the Friedel model is

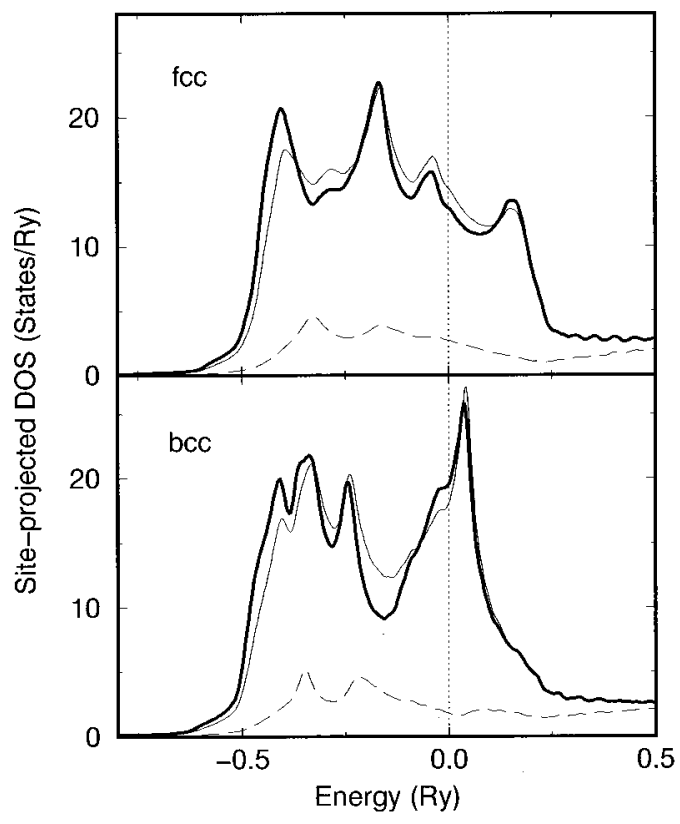

FIG. 5. State density for Ru in the fcc and bcc structures projected onto a vacancy site (broken line), onto an atomic site that is the nearest neighbor of the vacancy (thin line), and onto a distant bulk site (heavy line).

applied to the formation of vacancies ${ }^{9}$ it does lead to a maximum at the center of a transition series but the absolute values of the energies obtained in the model are $\sim 50 \%$ too large. The simple approach may be corrected by a pair potential and one then arrives at a model the parameters of which may be found by a fit to the measured vacancy formation energies. ${ }^{9,10}$ Thereby one confirms the empirical correlation, ${ }^{19}$

$$
E_{1 V}^{F} \approx \frac{1}{3} E_{c o h}^{\prime}
$$

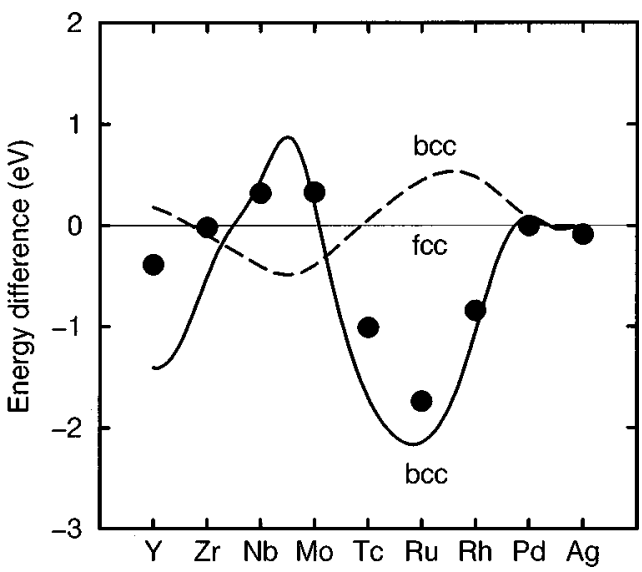

FIG. 6. bcc-fcc vacancy formation energy difference for the $4 d$ metals as calculated within the LSGF ASA $+M$ method, filled circles. The solid line shows the one-electron energy contribution obtained in a canonical band model, see text. The broken line shows the calculated structural energy difference curve. 
between the cohesive energy $E_{c o h}^{\prime}$ relative to a non-spinpolarized atomic state and the vacancy formation energy. Since $E_{c o h}^{\prime}$ varies parabolically with the $d$ occupation number, see Ref. 54, so does the vacancy formation energy.

\section{Crystal structure effects}

In Fig. 4 we show the monovacancy formation energies for the transition and noble metals calculated in the paramagnetic state assuming two cubic crystal structures. In the fcc structure each of the three series exhibits the parabolic behavior predicted by the Friedel model but with the maximum displaced to a $d$-band filling of $70 \%$, while in the bcc structure the monovacancy formation energy shows a pronounced minimum at the same $70 \% d$-band filling. We note that in the extreme cases, i.e., Fe, Ru, and Os, the structural contribution is a sizable fraction of the monovacancy formation energy and approximately a factor of 3 larger than the corresponding structural energy differences. ${ }^{48}$

To understand the origin of this large structural contribution we may use the local bond picture ${ }^{55}$ to construct the following simple model. Assume that the cohesive energy per atom may be written in the form

$$
\varepsilon_{c o h}^{\alpha}=\varepsilon_{c o h}^{\text {Fried }}+\varepsilon_{s t r}^{\alpha},
$$

where $\varepsilon_{c o h}^{\text {Fried }}$ is the energy of a reference state that we take to be the one-electron energy corresponding to Friedel's rectangular state density and $\varepsilon_{\text {str }}^{\alpha}$ is the structural contribution corresponding to the difference between the reference state density and the actual $\alpha$-structure state density.

At the formation of a vacancy the atoms next to it are perturbed and, as a result, the corresponding local state densities are rescaled, i.e, their bandwidths are reduced, and reshaped. Both effects are clearly seen in Fig. 5, where we show the relevant state densities for $\mathrm{Ru}$ in the two cubic crystal structures. For simplicity we will assume that only the atoms closest to the vacancy are perturbed and, hence, that only these atoms contribute to the vacancy formation energy. As will be shown below by means of Andersen's canonical band picture ${ }^{56}$ this assumption leads to an accurate description of the vacancy formation energy for a $d$ transition-metal series.

In a tight-binding picture the decrease in the $d$ bandwidth caused by the reduction in the effective coordination number gives rise to a factor of $\sqrt{(z-1) / z}$, where $z$ is the bulk coordination number, in all the contributions to Eq. (15). This means that each of the $z$ perturbed atoms contributes [ 1 $-\sqrt{(z-1) / z}] \varepsilon_{c o h}^{\alpha}$ to the vacancy formation energy due to the rescaling of the state density. In addition, the lowering of the local symmetry around a vacancy tends to wash out some of the prominent features found in the local state density for the ideal crystal. This reshaping effect gives rise to an additional contribution to the vacancy formation energy that stems from $\varepsilon_{\text {str }}^{\alpha}$ and has the prefactor $\sqrt{(z-1) / z}$.

With the above assumptions the final expression for the vacancy formation energy becomes

$$
E_{v a c}^{\alpha}=z\left(1-\sqrt{\frac{z-1}{z}}\right) \varepsilon_{c o h}^{\alpha}+z \sqrt{\frac{z-1}{z}} \Delta \varepsilon_{s t r}^{\alpha} .
$$

Here, the first term is, in fact, Eq. (14) and it includes a structural contribution that will add to the difference in the vacancy formation energy of the fcc and bcc structures. This structural contribution is, however, greatly reduced on account of its coordination number dependent prefactor, and cannot explain the structural effects seen in Fig. 4.

The second term in Eq. (16) represents the effect of reshaping the state density for a given crystal structure, i.e., it is the change in the stuctural contribution to the cohesive energy that is caused by the vacancy formation. Although the value of $\Delta \varepsilon_{s t r}^{\alpha}$ is not known in general, it is clear that in the "featureless" limit, where the reshaping effect is so strong that the atoms next to the vacancy have a rectangular state density, one has $\Delta \varepsilon_{s t r}^{\alpha}=-\varepsilon_{s t r}^{\alpha}$. Further, in contrast to the first term in Eq. (16), this structural contribution is enhanced by a factor of the order of the coordination number $z$. It can therefore lead to a large difference in the vacancy formation energy of the fcc and bcc structures.

We may now use our model to explain the vacancy formation energies shown in Fig. 4. For a transition-metal series we find that the first term in Eq. (16) represents essentially a parabolic variation with the $d$ occupation number leading to only a small structural difference in the vacancy formation energies for the two cubic structures while the second term leads to the large reduction in the vacancy formation energies seen in the (paramagnetic) bcc metals $\mathrm{Mn}, \mathrm{Fe}, \mathrm{Co}, \mathrm{Tc}$, $\mathrm{Ru}, \mathrm{Rh}, \mathrm{Re}, \mathrm{Os}$, and Ir. It follows from the outline of the model that the origin of this effect is the reduction of the pseudogap of the bcc state density upon vacancy formation that is the prominent feature responsible for the stability of the bcc structure for a half-filled $d$ band.

The onset of magnetism leads to a reduction in the surface energy of the magnetic $3 d$ metals described, for instance, by a spin-polarized Friedel model. ${ }^{57}$ As a result, one might expect a similar reduction for the vacancy formation energy of ferromagnetic Fe. However, in this case one finds an increase of about $0.95 \mathrm{eV}$ when going from paramagnetic to ferromagnetic bcc Fe, cf. Figs. 2 and 4, and Table IV. The reason for this increase is easily traced to the structural contribution given by the second term in Eq. (16). In the paramagnetic case bcc Fe has a low vacancy formation energy as a result of the large structural contribution for $d$ occupation numbers close to 7. However, in the completely saturated spinpolarized case bcc Fe is described by a minority-spin $d$ occupation number close to that of bcc $\mathrm{Cr}$ where the structural contribution is small and therefore ferromagnetic bcc Fe is predicted to have a vacancy formation energy close to that of $\mathrm{Cr}$, as indeed it has. The increase may therefore be viewed as a structural effect.

The simple analysis above may be quantified if we apply the canonical band approach to the total supercell $\mathrm{Ru}$ statedensities $D_{i}$ and calculate the first-order energy moments

$$
E_{i}=\int^{E_{F}}\left(\varepsilon-C_{i}\right) D_{i}(\varepsilon) d \varepsilon
$$


TABLE V. Calculated equilibrium atomic volume $\Omega_{0}$ in units of bohrs ${ }^{3}$, calculated and experimental relative vacancy formation volume, $\Omega_{1 V}^{F} / \Omega_{0}$.

\begin{tabular}{|c|c|c|c|c|c|c|}
\hline \multirow[t]{3}{*}{ Metal } & \multicolumn{3}{|c|}{ fcc } & \multicolumn{3}{|c|}{ bcc } \\
\hline & \multirow[t]{2}{*}{$\Omega_{0}$} & \multicolumn{2}{|c|}{$\Omega_{1 V}^{F} / \Omega_{0}$} & \multirow[t]{2}{*}{$\Omega_{0}$} & \multicolumn{2}{|c|}{$\Omega_{1 V}^{F} / \Omega_{0}$} \\
\hline & & $\begin{array}{l}\text { This } \\
\text { work }\end{array}$ & $\begin{array}{l}\text { Experiment or } \\
\text { FP calculation }\end{array}$ & & $\begin{array}{l}\text { This } \\
\text { work }\end{array}$ & $\begin{array}{l}\text { Experiment or } \\
\text { FP calculation }\end{array}$ \\
\hline $\mathrm{Sc}$ & 155 & 0.73 & & 160 & 0.69 & \\
\hline $\mathrm{Ti}$ & 111 & 0.68 & & 110 & 0.69 & \\
\hline $\mathrm{V}$ & 87 & 0.67 & & 83 & 0.64 & \\
\hline $\operatorname{Cr}(p)$ & 75 & 0.64 & & 72 & 0.79 & \\
\hline $\operatorname{Cr}(a)$ & & & & 72 & 0.71 & \\
\hline $\operatorname{Mn}(p)$ & 68 & 0.68 & & 67 & 0.59 & \\
\hline $\operatorname{Mn}(f)$ & 68 & 0.72 & & & & \\
\hline $\mathrm{Fe}(p)$ & 64 & 0.70 & & 65 & 0.63 & \\
\hline $\operatorname{Fe}(f)$ & & & & 69 & 0.55 & $0.95^{\mathrm{a}}$ \\
\hline $\operatorname{Co}(p)$ & 64 & 0.70 & $0.95^{\mathrm{a}}$ & 65 & 0.67 & \\
\hline $\operatorname{Co}(f)$ & 67 & 0.66 & & & & \\
\hline $\mathrm{Ni}(p)$ & 66 & 0.71 & $0.80^{\mathrm{a}}$ & 66 & 0.70 & \\
\hline $\mathrm{Ni}(f)$ & 67 & 0.72 & & & & \\
\hline $\mathrm{Cu}$ & 72 & 0.70 & $0.75,^{\mathrm{a}} 0.70^{\mathrm{b}}$ & 72 & 0.71 & \\
\hline $\mathrm{Y}$ & 206 & 0.76 & & 212 & 0.81 & \\
\hline $\mathrm{Zr}$ & 151 & 0.70 & $0.95^{\mathrm{a}}$ & 146 & 0.78 & \\
\hline $\mathrm{Nb}$ & 120 & 0.60 & & 113 & 0.74 & \\
\hline Mo & 103 & 0.60 & & 100 & 0.65 & $0.90,^{\mathrm{a}} 0.60^{\mathrm{c}}$ \\
\hline $\mathrm{Tc}$ & 94 & 0.66 & & 93 & 0.66 & \\
\hline $\mathrm{Ru}$ & 89 & 0.71 & & 90 & 0.66 & \\
\hline $\mathrm{Rh}$ & 89 & 0.73 & & 91 & 0.66 & \\
\hline $\mathrm{Pd}$ & 96 & 0.68 & & 95 & 0.70 & \\
\hline $\mathrm{Ag}$ & 108 & 0.76 & & 107 & 0.78 & \\
\hline $\mathrm{Lu}$ & 190 & 0.80 & & 196 & 0.72 & \\
\hline $\mathrm{Hf}$ & 140 & 0.76 & & 136 & 0.78 & \\
\hline $\mathrm{Ta}$ & 120 & 0.71 & & 114 & 0.76 & \\
\hline W & 106 & 0.63 & & 103 & 0.72 & \\
\hline $\mathrm{Re}$ & 97 & 0.66 & & 96 & 0.66 & \\
\hline Os & 93 & 0.72 & & 94 & 0.66 & \\
\hline $\mathrm{Ir}$ & 94 & 0.67 & & 96 & 0.67 & \\
\hline $\mathrm{Pt}$ & 100 & 0.69 & & 99 & 0.75 & \\
\hline $\mathrm{Au}$ & 113 & 0.73 & $0.85^{\mathrm{a}}$ & 111 & 0.72 & \\
\hline
\end{tabular}

aExperiment, Ref. 3.

${ }^{\mathrm{b}}$ FP-KKR-GF, Ref. 51.

${ }^{\mathrm{c}}$ Pseudopotential, Ref. 36.

for $i$ corresponding to the fcc and bcc supercells with and without vacancies as functions of the zeroth-order energy moments,

$$
n_{i}=\frac{1}{N_{a}} \int^{E_{F}} D_{i}(\varepsilon) d \varepsilon,
$$

i.e., the one-electron energies as functions of the occupation numbers per atom. Thereby, we obtain four one-electron energies whose proper combination yields the vacancy formation energy as a function of the zeroth-order moment, i.e., the $d$ occupation number.

In Fig. 6 we show the results of the canonical procedure where the first-order moments have been obtained relative to the centers of gravity $C_{i}$ corresponding to the total state density of $\mathrm{Ru}$ occupied by 11 electrons per atom for each of the four cases involved. The comparison with the results of the full calculation and the analysis above shows that the structural contribution to the vacancy formation energy can be traced to the well-known structural energy difference curve for $d$-band metals.

\section{VACANCY FORMATION VOLUME}

The calculated equilibrium atomic volumes and vacancy formation volumes are presented in Table V. We find that in all cases the vacancy formation volume is smaller than the atomic volume by approximately the same amount, i.e., $\Omega_{1 V}^{F} / \Omega_{0} \approx 0.7$, independent of crystal structure.

There is a dearth of experimental data on the vacancy formation volumes in transition metals. Moreover, even for 
the same metal-vacancy system the existing experimental data for the vacancy formation volumes are typically scattered within the range from $0.5 \Omega_{0}$ to $\Omega_{0}$. However, what appears to be the most reliable low-temperature data for the volume misfits as obtained by positron annihilation experiments have been tabulated in the Landolt-Börnstain reference series book ${ }^{3}$ from which the values of vacancy formation volumes can be deduced (see Table V).

Since the vacancy formation volume is a thermodynamic quantity, it reflects the overall relaxation of the volume of a crystal containing vacancies. This global volume relaxation of the crystal lattice is included in our calculations. It is clear, that any additional lattice relaxation, such as local relaxations around a vacancy site, will decrease the absolute value of the partial vacancy pressure and the volume misfit. Accordingly, the vacancy formation volume should approach $\Omega_{0}$ if local relaxations are present in the system. As a result, the present calculations, which neglect such relaxations, tend to underestimate the vacancy formation volumes in transition metals, and the small difference between our results and experiment may be attributed to the effects of local relaxation.

To our knowledge, there exist only two first-principles calculations of vacancy formation volumes. Papanikolaou et $a l .{ }^{51}$ used the FP-KKR Green's-function method for an isolated vacancy in $\mathrm{Cu}$ and calculated the volume misfit through the Kanzaki forces. The result of our calculation for the vacancy formation volume in $\mathrm{fcc} \mathrm{Cu}$ is in agreement with their result, $\delta=-0.3$ (see Table V). The vacancy formation volume for bcc Mo was calculated by Meyer and Fähnle ${ }^{36}$ using a 54-site supercell by means of a mixed-basis pseudopotential method. The difference between their result, $\Omega_{1 V}^{F} / \Omega_{0}$ $=0.6 \pm 0.1$, and our result (see Table $\mathrm{V}$ ) is within the expected error limits of both calculations.

\section{CONCLUSIONS}

We have used the order- $N$ LSGF method in conjunction with an electrostatic multipole correction to the atomicsphere approximation, ASA $+\mathrm{M}$, to study the monovacancy formation energies in the $3 d, 4 d$, and $5 d$ transition and noble metals. The results are in excellent agreement with the available full-potential calculations and with the recently "recommended" monovacancy formation energies for $\mathrm{Ni}$, $\mathrm{Cu}, \mathrm{Ag}, \mathrm{Pt}$, and $\mathrm{Au}$. For the remaining transition metals the calculations describe the trends observed experimentally, but owing to large uncertainties in the experimental values it appears that the calculated results at present form the most consistent estimate of the monovacancy formation energies in the three transition-metal series.

Note added. Since the completion of the present manuscript we have become aware of three recent publications related to the present paper: (i) a critical review of experimental results (Ref. 58); (ii) a study of the effect of the generalized gradient approximation on the vacancy formation energy (Ref. 59); (iii) an ab initio calculation of the vacancy formation and migration energy in W (Ref. 60).

\section{ACKNOWLEDGMENTS}

We are grateful to Dr. S. I. Simak and Dr. L. Vitos for supplying us with their computer codes for supercell construction and Madelung matrix calculation, respectively. One of us (P.A.K.) would like to thank Dr. N. M. Rosengaard and Dr. A. Yu. Lozovoi for useful discussions and comments. This work was partially supported by SKB AB, the Swedish Nuclear Fuel and Waste Management Company. The support by the Swedish Natural Science Research Council is acknowledged. Center for Atomic-Scale Materials Physics is sponsored by the Danish National Research Foundation.
*Also at the Department of Theoretical Physics, Moscow State Institute of Steel and Alloys, Russia.

${ }^{1}$ H.-E. Schaefer, Phys. Status Solidi A 102, 47 (1987).

${ }^{2}$ R. W. Siegel, in Proceedings of the 6th International Conference on Positron Annihilation, edited by P. G. Coleman, S. C. Sharma, and L. H. Diaena (North-Holland, Amsterdam, 1982), p. 351.

${ }^{3}$ P. Ehrhart, P. Jung, H. Schultz, and H. Ullmaier, in Atomic Defects in Metals, edited by H. Ullmaier, Landolt-Börnstein, New Series, Group III, Vol. 25 (Springer-Verlag, Berlin, 1991).

${ }^{4}$ M. J. Gillan, J. Phys.: Condens. Matter 1, 689 (1989).

${ }^{5}$ W. Frank, U. Breier, C. Elsässer, and M. Fähnle, Phys. Rev. B 48, 7676 (1993).

${ }^{6}$ N. Chetty, M. Weinert, T. S. Rahman, and J. W. Davenport, Phys. Rev. B 52, 6313 (1995).

${ }^{7}$ B. Drittler, M. Weinert, R. Zeller, and P. H. Dederichs, Solid State Commun. 79, 31 (1991).

${ }^{8}$ P. H. Dederichs, B. Drittler, and R. Zeller, in Application of Multiple Scattering Theory to Materials Science, edited by W. H. Butler et al., MRS Symposia Proceedings No. 253 (Materials Research Society, Pittsburgh, 1992), p. 185.

${ }^{9}$ H. M. Polatoglou, M. Methfessel, and M. Scheffler, Phys. Rev. B 48, 1877 (1993)

${ }^{10}$ T. Korhonen, M. J. Puska, and R. M. Nieminen, Phys. Rev. B 51, 9526 (1995).

${ }^{11}$ U. Klemradt, B. Drittler, R. Zeller, and P. H. Dederichs, Phys. Rev. Lett. 64, 2803 (1990).
${ }^{12}$ N. Stefanou, N. Papanikolaou, and P. H. Dederichs, J. Phys.: Condens. Matter 3, 8793 (1991).

${ }^{13}$ U. Klemradt, B. Drittler, T. Hoshino, R. Zeller, P. H. Dederichs, and N. Stefanou, Phys. Rev. B 43, 9487 (1991).

${ }^{14}$ T. Hoshino, R. Zeller, P. H. Dederichs, and M. Wienert, Europhys. Lett. 24, 495 (1993).

${ }^{15}$ T. Hoshino, R. Zeller, and P. H. Dederichs, Phys. Rev. B 53, 8971 (1996).

${ }^{16}$ M. Lannoo and G. Allan, J. Phys. Chem. Solids 32, 637 (1971).

${ }^{17}$ F. R. de Boer, R. Boom, W. C. M. Mattens, A. R. Miedema, and A. K. Niessen, in Cohesion in Metals: Transition Metal Alloys, edited by F. R. de Boer and D. G. Pettifor (North-Holland, Amsterdam, 1988), Vol. 1.

${ }^{18}$ M. J. Mehl and D. A. Papaconstantopoulos, Phys. Rev. B 54, 4519 (1996).

${ }^{19}$ T. Górecki, Z. Metallkd. 65, 426 (1974).

${ }^{20}$ P. R. Couchman, Phys. Lett. 54A, 309 (1975).

${ }^{21}$ J. P. Perdew, Y. Wang, and E. Engel, Phys. Rev. Lett. 66, 508 (1991).

${ }^{22}$ J. A. Alonso and N. H. March, Electrons in Metals and Alloys (Academic Press, London, 1989).

${ }^{23}$ G. Grimvall and S. Sjödin, Phys. Scr. 10, 340 (1974).

${ }^{24}$ C. L. Reynolds, Jr. and P. R. Couchman, Phys. Lett. 50A, 157 (1974).

${ }^{25}$ D. M. C. Nicholson, G. M. Stocks, Y. Wang, W. A. Shelton, Z. Szotek, and W. M. Temmerman, Phys. Rev. B 50, 14686 (1994). 
${ }^{26}$ Y. Wang, G. M. Stocks, W. A. Shelton, D. M. C. Nicholson, Z. Szotek, and W. M. Temmerman, Phys. Rev. Lett. 75, 2867 (1995).

${ }^{27}$ I. A. Abrikosov, A. M. N. Niklasson, S. I. Simak, B. Johansson, A. V. Ruban, and H. L. Skriver, Phys. Rev. Lett. 76, 4203 (1996).

${ }^{28}$ I. A. Abrikosov, S. I. Simak, B. Johansson, A. V. Ruban, and H. L. Skriver, Phys. Rev. B 56, 9319 (1997).

${ }^{29}$ T. Beuerle, R. Pawellek, C. Elsässer, and M. Fähnle, J. Phys.: Condens. Matter 3, 1957 (1991).

${ }^{30}$ P. Braun, M. Fähnle, M. van Schilfgaarde, and O. Jepsen, Phys. Rev. B 44, 845 (1991).

${ }^{31}$ M. Sinder, D. Fuks, and J. Pelleg, Phys. Rev. B 50, 2775 (1994).

${ }^{32}$ H. L. Skriver and N. M. Rosengaard, Phys. Rev. B 43, 9538 (1991).

${ }^{33}$ A. V. Ruban, I. A. Abrikosov, D. Ya. Kats, D. Gorelikov, K. W. Jacobsen, and H. L. Skriver, Phys. Rev. B 49, 11383 (1994).

${ }^{34}$ I. A. Abrikosov, A. V. Ruban, H. L. Skriver, and B. Johansson, Phys. Rev. B 50, 2039 (1994).

${ }^{35}$ Here we consider the vacancy formation energy at a fixed volume per site. The thermodynamically consistent definition requires that not only the total volume of the system $V$ but also the number of atoms $N_{a}$ be kept fixed. Under these constraints, the partial vacancy formation pressure may be defined as $p_{1 V}^{F}$ $=\left(\partial p / \partial c_{v}\right)_{V_{0}}=B \Omega_{1 V}^{F} / \Omega_{0}$. This pressure is always positive, in contrast to $p_{1 V}$.

${ }^{36}$ B. Meyer and M. Fähnle, Phys. Rev. B 56, 13595 (1997).

${ }^{37}$ O. K. Andersen, Phys. Rev. B 12, 3060 (1975).

${ }^{38}$ O. Gunnarsson, O. Jepsen, and O. K. Andersen, Phys. Rev. B 27, 7144 (1983).

${ }^{39}$ H. L. Skriver, The LMTO Method (Springer-Verlag, Berlin, 1984).

${ }^{40}$ O. K. Andersen and O. Jepsen, Phys. Rev. Lett. 53, 2571 (1984).

${ }^{41}$ O. K. Andersen, O. Jepsen, and D. Glötzel, in Highlights of Condensed-Matter Theory, edited by F. Bassani, F. Fumi, and
M. P. Tosi (North-Holland, New York, 1985).

${ }^{42}$ O. K. Andersen, Z. Pawlowska, and O. Jepsen, Phys. Rev. B 34, 5253 (1986).

${ }^{43}$ L. Vitos, J. Kollàr, and H. L. Skriver, Phys. Rev. B 55, 13521 (1997).

${ }^{44}$ E. Esposito, A. E. Carlsson, D. D. Ling, H. Ehrenreich, and C. D. Gelatt, Jr., Philos. Mag. A 41, 251 (1980).

${ }^{45}$ D. Glötzel and O. K. Andersen (unpublished).

${ }^{46}$ A. K. McMahan, H. L. Skriver, and B. Johansson, Phys. Rev. B 23, 5016 (1981).

${ }^{47}$ N. E. Christensen and S. Satpathy, Phys. Rev. Lett. 55, 600 (1985).

${ }^{48}$ H. L. Skriver, Phys. Rev. B 31, 1909 (1985).

${ }^{49}$ J. P. Perdew, K. Burke, and M. Ernzerhof, Phys. Rev. Lett. 77, 3865 (1996).

${ }^{50}$ D. M. Ceperley and B. J. Alder, Phys. Rev. Lett. 45, 566 (1980).

${ }^{51}$ N. Papanikolaou, R. Zeller, P. H. Dederichs, and N. Stefanon, Phys. Rev. B 55, 4157 (1997).

${ }^{52}$ L. de Schepper, D. Segers, L. Dorikens-Vanpraet, M. Dorikens, G. Knuyt, L. M. Stals, and P. Moser, Phys. Rev. B 27, 5257 (1983).

${ }^{53} \mathrm{~J}$. Friedel, in The Physics of Metals, edited by J. M. Ziman (Cambridge University Press, Cambridge, 1969), p. 494.

${ }^{54}$ M. S. S. Brooks and B. Johansson, J. Phys. F 13, L197 (1983).

${ }^{55}$ A. V. Ruban, H. L. Skriver, and J. K. Nørskov, Phys. Rev. Lett. 80, 1240 (1998).

${ }^{56}$ O. K. Andersen, J. Madsen, U. K. Poulsen, O. Jepsen, and J. Kollar, Physica B \& C 86-88B, 249 (1977).

${ }^{57}$ M. Alden, H. L. Skriver, S. Mirbt, and B. Johansson, Phys. Rev. Lett. 69, 2296 (1992).

${ }^{58}$ Y. Kraftmakher, Phys. Rep. 299, 79 (1998).

${ }^{59}$ M. Asato, T. Hoshino, T. Asada, R. Zeller, and P. H. Dederichs, J. Magn. Magn. Mater. 177-181, 1403 (1998).

${ }^{60}$ A. Satta, F. Willaime, and S. de Gironcoli, Phys. Rev. B 57, 11 184 (1998) 